$\mathrm{J}$ o u r n a l of

Mathematics

and Applications

JMA No 44, pp 57-70 (2021)

\title{
Measures of Growth and Approximation of Entire Harmonic Functions in $n$-Dimensional Space in Some Banach Spaces
}

\author{
Devendra Kumar
}

\begin{abstract}
The relationship between the classical order and type of an entire harmonic function in space $\mathbb{R}^{n}, n \geq 3$, and the rate of its best harmonic polynomial approximation for some Banach spaces of functions harmonic in the ball of radius $R$ has been studied.
\end{abstract}

AMS Subject Classification: 30E10, 41A15.

Keywords and Phrases: Entire harmonic function, Approximation errors, Banach spaces, order and type, Gegenbauer polynomials and spherical harmonics.

\section{Introduction}

Several authors like Vakarchuk [24], Vakarchuk and Zhir [25,26], Srivastava and Kumar [20], Harfaoui [7] and others have studied the growth parameters of an entire function in terms of coefficients occurring in its Taylor series expansion and polynomial approximation errors in some Banach spaces. Since entire harmonic functions play an important role in physics and mechanics to describe different stationary processes and in mathematical research, it is significant to study the growth characteristics order and type of entire harmonic functions in terms of coefficients occurring in its Fourier-Laplace series [23] and harmonic polynomial approximation errors in space $\mathbb{R}^{n}, n \geq 3$ in some Banach spaces. To the best of our knowledge this study has not been done so far. In this paper our aim is to bridge this gap.

A number of papers $[3,4,10-17,19,21]$ were devoted to establishing a relation between

COPYRIGHT (C) by Publishing House of Rzeszów University of Technology P.O. Box 85, 35-959 Rzeszów, Poland 
the growth of entire harmonic functions in $\mathbb{R}^{n}, n \geq 3$ and the behavior of expansion coefficients, spherical harmonics and harmonic polynomial approximation errors. In particular, when we discuss time dependent problems in $\mathbb{R}^{3}$ it leads to study the harmonic functions in $\mathbb{R}^{4}$. Therefore, to study the entire harmonic functions in $\mathbb{R}^{n}, n \geq 3$ is reasonable.

Let $x \in \mathbb{R}^{n}(n \geq 3)$ be an arbitrary point where $x=\left(x_{1}, x_{2}, \ldots, x_{n}\right)$ and put $|x|=\left(x_{1}^{2}+x_{2}^{2}+\cdots+x_{n}^{2}\right)^{\frac{1}{2}}$. The set of all non-constant entire harmonic functions on $\mathbb{R}^{n}$ is denoted by $H$. For each $u \in H, r>0$, the Fourier-Laplace series expansion of $u$ be given as [23]

$$
u(r x)=\sum_{k=0}^{\infty} Y^{(k)}(x ; u) r^{k}
$$

where $x \in S^{n}=\left\{x \in \mathbb{R}^{n}:|x|=1\right\}$ a unit sphere in $\mathbb{R}^{n}$ centered at the origin and

$$
\begin{gathered}
Y^{(k)}(x ; u)=a_{1}^{(k)} Y_{1}^{(k)}(x)+a_{2}^{(k)} Y_{2}^{(k)}(x)+\cdots+a_{\gamma_{k}}^{(k)} Y_{\gamma_{k}}^{(k)}(x), \\
a_{j}^{(k)}=\left(u, Y_{j}^{(k)}\right)=\frac{\Gamma(n / 2)}{2(\pi)^{\frac{n}{2}}} \int_{S^{n}} u(x) Y_{j}^{(k)}(x) d S, j=\overline{1, \gamma_{k}}, \\
\gamma_{k}=\frac{(2 k+n-2)(k+n-3) !}{k !(n-2) !} .
\end{gathered}
$$

Here $d S$ is the element of the surface area on the sphere $S^{n},\left(u, Y_{j}^{(k)}\right)$ is the scalor product in $L^{2}\left(S^{n}\right)$ and $Y^{(k)}$ is a spherical harmonic of degree $k, k \in Z_{+}=\{0,1,2, \ldots$, on the unit sphere $S^{n}(n \geq 2)[22]$.

Let $B_{R}^{n}=\left\{y \in \mathbb{R}^{n}:|y| \leq R\right\}$ be the ball of radius $\mathrm{R}$ in space $\mathbb{R}^{n}, n \geq 3$ centered at the origin, and $\overline{B_{R}^{n}}$ be the closure of $B_{R}^{n}$. We denote $H_{R}$, the class of harmonic functions in $B_{R}^{n}$ and continuous on $\overline{B_{R}^{n}}, 0<R<\infty$.

We now consider some of the Banach spaces.

1. The space $B$ of functions harmonic in the ball $B_{R}^{n}$ and continuous on $\overline{B_{R}^{n}}$ i.e., $u \in H_{R}$ with norm $\|u\|=\max _{y \in \overline{B_{R}^{n}}}|u(y)|<\infty$.

2. The Hardy spaces $H_{p}, p \geq 1$, of functions harmonic in the ball $B_{R}^{n}$ with norm

$$
\|u\|_{H_{p}}=\sup _{0<r<R} M_{p}(r ; u), M_{p}(r ; u)=\left(\frac{1}{(2 \pi)^{n}} \int_{T^{n}}\left|u\left(r e^{i t} x\right)\right|^{p} d t\right)^{\frac{1}{p}}, p \in[1, \infty),
$$

where $T^{n}=\left\{x \in \mathbb{R}^{n}: 0 \leq x_{j} \leq 2 \pi, j=\overline{1, n}\right\}$,

$\|u\|=\sup _{y \in B_{R}^{n}}|u(y)|, p=\infty$.

3. The Bergman spaces $H_{p}^{\prime}$ of functions harmonic in the ball $B_{R}^{n}$ for $p \in[1, \infty)$ with the norm

$$
\|u\|_{H_{p}^{\prime}}=\left(\frac{1}{(\pi)^{n}} \int_{S^{n}}|u(r x)|^{p} d x\right)^{\frac{1}{p}}
$$


4. The spaces $A_{p}, p \in(0,1)$ of functions harmonic in the ball $B_{R}^{n}$ with norm

$$
\|u\|_{A_{p}}=\int_{S^{n}}\left(\frac{R-r}{R}\right)^{\frac{1}{p}-2} M_{1}(r ; u) d r .
$$

5. The spaces $B_{p, q, \lambda}, 0<p<q \leq \infty, \lambda>0$, of functions harmonic in the ball $B_{R}^{n}$ with the norm

$$
\|u\|_{p, q, \lambda}=\left\{\int_{S^{n}}\left(\frac{R-r}{R}\right)^{\lambda\left(\frac{1}{p}-\frac{1}{q}\right)-1} M_{q}^{\lambda}(r ; u) d r\right\}^{\frac{1}{\lambda}}, \lambda<\infty,
$$

and

$$
\|u\|_{p, q, \infty}=\sup _{0<r<R}\left\{\left(\frac{R-r}{R}\right)^{\left(\frac{1}{p}-\frac{1}{q}\right)} M_{q}(r ; u)\right\}, \lambda=\infty,
$$

for $\min (q, \lambda) \geq 1, B_{p, q, \lambda}$ are Banach spaces.

We denote a Banach space $X$ formed by the functions harmonic in $B_{R}^{n}$ with finite norm $\|$.$\| given by (1-5).$

An approximation error of function $u \in H_{R}$ by harmonic polynomials $P \in \Pi_{k}$ is defined as

$$
E_{R}^{k}(u)=\inf \left\{\|u(y)-P(y)\|, y \in \overline{B_{R}^{n}}\right.
$$

where $\Pi_{k}$ be a set of harmonic polynomials of degree not exceeding $k$.

The relationship between the order and type of an entire function $f$ in terms of the sequence $E_{R}^{k}(f)$ in the space $H_{2}^{\prime}$ were obtained in [19] and for the spaces $H_{p}^{\prime}, p \geq 1$ were studied by Ibragimov and Shikhaliev $[8,9]$. The spaces $A_{p}, p \in(0,1)$ of functions analytic in the unit disk were first studied by Hardy and Littlewood [6] and later by Romberg, Duren and Shields [2]. The spaces $B_{p, q, \lambda}, 0<p<q \leq \infty, \lambda>0$ were considered in $[5,6]$. The order and type of entire functions in terms of approximation errors $E_{R}^{k}(f)$ in the spaces $B_{p, q, \lambda}$ were obtained by Vakarchuk [24].

\section{Auxiliary Results}

Lemma 2.1. Let $u \in X$ and $u(\tau x)=\sum_{k=0}^{\infty} Y^{k}(x ; u) \tau^{k}, 0<\tau<R$, be an entire harmonic function in $\mathbb{R}^{n}$. Then

$$
\lim _{k \rightarrow \infty}\left\{\frac{\left\|\tau^{k}\right\|_{X}}{R^{k}}\right\}^{\frac{1}{k}}=1
$$

Proof. For an entire harmonic function $u$ in the space $B$ and $H_{p}, 0<p \leq \infty$, respectively, the quantity $\left\|\tau^{k}\right\|_{X}, k \in \mathbb{Z}_{+}$is

$$
\left\|\tau^{k}\right\|_{X}=R^{k}
$$


it gives (2.1).

In the space $X=H_{p}^{\prime}, p \geq 1$, we have

$$
\frac{\left\|\tau^{k}\right\|_{H_{p}^{\prime}}}{R^{k}}=(k p+2)^{\frac{1}{p^{2}}} \leq\left\{k p\left(1+\frac{2}{k p}\right)\right\}^{\frac{1}{p^{2}}}, k \geq 0
$$

or

$$
\frac{\left\|\tau^{k}\right\|_{H_{p}^{\prime}}}{R^{k}} \leq \chi_{H_{p}^{\prime}} k^{\frac{1}{p^{2}}}
$$

where $\chi_{H_{p}^{\prime}}=p^{\frac{1}{p^{2}}}\left(1+\frac{1}{p}\right)^{\frac{1}{p^{2}}}$.

From (2.3) we obtain the following upper bound

$$
\lim _{k \rightarrow \infty}\left\{\frac{\left\|\tau^{k}\right\|_{H_{p}^{\prime}}}{R^{k}}\right\}^{\frac{1}{k}} \leq 1 .
$$

For lower bound using (2.2) and we get

$$
\frac{\left\|\tau^{k}\right\|_{H_{p}^{\prime}}}{R^{k}} \geq p^{\frac{1}{p^{2}}} k^{\frac{1}{p^{2}}} \geq(p k)^{\frac{1}{p^{2}}}
$$

or

$$
\lim _{k \rightarrow \infty}\left\{\frac{\left\|\tau^{k}\right\|_{H_{p}^{\prime}}}{R^{k}}\right\}^{\frac{1}{k}} \geq 1
$$

Combining (2.4) and (2.5) we get the required result.

In the space $X=A_{p}, 0<p<1$, we have

$$
\frac{\left\|\tau^{k}\right\|_{A_{p}}}{R^{k}}=(2 \pi)^{-\frac{1}{p}}\left(B\left(k p+1 ; \frac{1}{p}-1\right)\right)^{-\frac{1}{p^{2}}} .
$$

The right hand side of (2.6) can be estimated by using the relation between the Euler integral of the first kind $B(a, b)$ and $\Gamma$ function for $a, b>0$,

$$
B(a, b)=\frac{\Gamma a \Gamma b}{\Gamma(a+b)}
$$

and the asymptotic relation

$$
\frac{\Gamma\left(\xi+s_{1}\right)}{\Gamma\left(\xi+s_{2}\right)}=\xi^{s_{1}-s_{2}}\left(1+\frac{\left(s_{1}-s_{2}\right)\left(s_{1}+s_{2}-1\right)}{2 x}+o\left(\left|\xi^{-2}\right|\right)\right.
$$

where $|\xi| \geq 1, \xi \in \mathbb{R}$ and $s_{1}$ and $s_{2}$ are arbitrary fixed real numbers.

Set $\xi=k p, s_{1}=\frac{1}{p}$ and $s_{2}=1$, for sufficiently large $k$, for $k \geq 1$ in above relations, we get

$$
\begin{aligned}
\frac{\left\|\tau^{k}\right\|_{A_{p}}}{R^{k}} & =\left[\frac{(2 \pi)^{-p} \Gamma\left(k p+\frac{1}{p}\right)}{\Gamma^{\frac{1}{p^{2}}}\left(\frac{1}{p}-1\right)(k p+1)}\right]^{\frac{1}{p^{2}}} \\
& =\frac{(2 \pi)^{-p} p^{\frac{1}{p^{2}\left(\frac{1}{p}-1\right)}}}{\left(\frac{1}{p}-1\right)}\left(k\left(1+\frac{\left(\frac{1}{p}-1\right) \frac{1}{p}}{2 k p}+O\left(k^{-2} p^{2}\right)\right)\right)^{\frac{1}{p^{2}}} \\
& \leq \chi_{A_{p}} k^{\frac{1}{p^{2}}}
\end{aligned}
$$


where

$$
\chi_{A_{p}}=\frac{(2 \pi)^{-p} p^{\frac{1}{p^{2}\left(\frac{1}{p}-1\right)}}}{\left(\frac{1}{p}-1\right)}\left(1+\left(\frac{1}{p}-1\right) \frac{1}{p}+A\right)^{\frac{1}{p^{2}}}
$$

here $A$ is an absolute constant independent of $k$.

$$
\lim _{k \rightarrow \infty}\left\{\frac{\left\|\tau^{k}\right\|_{A_{p}}}{R^{k}}\right\}^{\frac{1}{k}} \leq 1
$$

For lower bound we have

$$
\frac{\left\|\tau^{k}\right\|_{A_{p}}}{R^{k}} \geq \frac{(2 \pi)^{-p} p^{\frac{1}{p^{2}\left(\frac{1}{p}-1\right)}}}{\left(\frac{1}{p}-1\right)} k^{\frac{1}{p^{2}}} \geq\left\{\frac{k}{\Gamma\left(\frac{1}{p}-1\right)}\right\}^{\frac{1}{p^{2}}}
$$

or

$$
\lim _{k \rightarrow \infty}\left\{\frac{\left\|\tau^{k}\right\|_{A_{p}}}{R^{k}}\right\}^{\frac{1}{k}} \geq 1
$$

Using (2.7) and (2.8) we get the required result.

Following on the lines of [26] for single complex variable we obtain for the space $X=B_{p, q, \lambda}, 0<p<q \leq \infty, 0<\lambda \leq \infty$ that

$$
\lim _{k \rightarrow \infty}\left\{\frac{\left\|\tau^{k}\right\|_{B_{p, q, \lambda}}}{R^{k}}\right\}^{\frac{1}{k}}=1 .
$$

Lemma 2.2. Let $u \in X$ and let

$$
u(\tau x)=\sum_{k=0}^{\infty} Y^{(k)}(x ; u) \tau^{k} \text { in space } \mathbb{R}^{n}, n \geq 3,0<\tau<R .
$$

Then

$\left|Y^{(k)}(x ; u)\right|\left\|\tau^{k}\right\|_{X} \leq \frac{2 \sqrt{2}(k+2 \nu)}{C \sqrt{(2 \nu) !}(2 \nu+1)(k-1+2 \nu)^{2 \nu}}\left(\frac{r}{R}\right)^{k-1} E_{R}^{k-1}(u) \leq\|u(\tau x)\|_{X}$,

where $C$ is a constant independent of $u$ and $\tau x$.

Proof. Using the addition theorem [1] for the Gegenbauer polynomials $C_{k}^{\nu}$ of degree $k$ and order $\nu$, we have

$$
\int_{S^{n}} C_{k}^{\nu}[(x, \zeta)] P(\tau \zeta) d S(\zeta)=0
$$

where $P \in \Pi_{k-1}, 0<\tau<R, x \in S^{n}, \zeta \in S^{n}$, and

$$
Y^{(k)}(x ; u) r^{k}=\frac{2(k+\nu)}{d_{n} w_{n}} \int_{S^{n}} C_{k}^{\nu}[(x, y)] u(r y) d S(y)
$$


where $k \in Z, d_{2}=1, d_{n}=n-2$ at $n>2, \nu=\frac{n-2}{2}, w_{n}=\frac{2(\pi)^{\frac{n}{2}}}{\Gamma\left(\frac{n}{2}\right)}$.

Rewrite (2.9) as

$$
Y^{(k)}(x ; u) \tau^{k}=\frac{(k+\nu)}{\nu w_{n}} \int_{S^{n}} C_{k}^{\nu}[(x, \zeta)][u(\tau \zeta)-P(\tau \zeta)] d S(\zeta)
$$

Since $\max _{-1 \leq t \leq 1}\left|C_{k}^{\nu}(t)\right|=C_{k}^{\nu}(1)$, from [1] we have $C_{k}^{\nu}(1)=\frac{(k+2 \nu-1) !}{\left(d_{n}-1\right) ! k !}$ and from $(2.10)$ we obtain

$$
\begin{aligned}
\left|Y^{(k)}(x ; u)\right|\left\|\tau^{k}\right\|_{X} \leq & \frac{(k+\nu)}{\nu w_{n}}\|u(\tau \zeta)-P(\tau \zeta)\|_{X} C_{k}^{\nu}(1) w_{n} \\
& \leq \frac{2(k+2 \nu)^{2 \nu}}{(2 \nu) !}\|u(\tau \zeta)-P(\tau \zeta)\|_{X}
\end{aligned}
$$

there exists a polynomial $P^{*} \in \Pi_{k-1}$ for which

$$
\|u(\tau \zeta)-P(\tau \zeta)\|_{X} \leq C \max \left|u(\tau \zeta)-P^{*}(\tau \zeta)\right| \leq 2 E_{R}^{k-1}(u)
$$

So we have

$$
\left|Y^{(k)}(x ; u)\right|\left\|\tau^{k}\right\|_{X} \leq \frac{4 C(k+2 \nu)^{2 \nu}}{(2 \nu) !} E_{R}^{k-1}(u)
$$

Now consider $Q(\tau \zeta)=\sum_{j=0}^{k} Y^{(j)}(\zeta ; u) \tau^{j}$, since $Q \in \Pi_{k}$, we have

$$
E_{R}^{k}(u) \leq\|u(\tau \zeta)-Q(\tau \zeta)\|_{X} \leq C \max _{\tau \zeta \in \overline{B_{R}^{n}}}|u(\tau \zeta)-Q(\tau \zeta)|
$$

Using a result of [27] we have

$$
\begin{aligned}
& E_{R}^{k}(u) \leq \sum_{j=k+1}^{\infty} C \max _{\zeta \in S^{n}}\left|Y^{(j)}(\zeta, u)\right| R^{j} \\
& \leq C \sqrt{\frac{2}{(2 \nu) !}}\|u(\tau \zeta)\|_{X} \sum_{j=k+1}^{\infty}(j+2 \nu)^{\nu}\left(\frac{R}{r}\right)^{j} \\
& =C \sqrt{\frac{2}{(2 \nu) !}}\|u(\tau \zeta)\|_{X}\left(\frac{R}{r}\right)^{k} \sum_{j=k+1}^{\infty}(j+2 \nu)^{\nu}\left(\frac{R}{r}\right)^{j-k} .
\end{aligned}
$$

For $r>e R$, the maximum value of last sum can be estimate as

$$
\sum_{j=k+1}^{\infty}(j+2 \nu)^{\nu}\left(\frac{R}{r}\right)^{j-k} \leq e^{k} \sum_{j=k+1}^{\infty}(j+2 \nu)^{\nu} e^{-j} \leq e^{k} \int_{k}^{\infty}(t+2 \nu)^{2 \nu} e^{-t} d t
$$


Set $\eta=2 \nu, \theta_{\eta}(t)=(t+\eta)^{\eta}$ and integrating $(\eta+1)$ times by parts, we get

$$
\begin{aligned}
\int_{k}^{\infty} \theta_{\eta}(t) e^{-t} d t= & {\left.\left[-e^{-t}\left(\theta_{\eta}(t)+\theta_{\eta}^{\prime}(t)+\cdots+\theta_{\eta}^{(n)}(t)\right)\right]\right|_{k} ^{\infty} } \\
& =e^{-k} \sum_{i=0}^{\eta} \frac{\eta !(k+\eta)^{\eta-i}}{(\eta-i) !}, \text { since } \theta_{\eta}^{(i)}=\frac{\eta !}{(\eta-i)}(t+\eta)^{\eta-i}, i=\overline{1, \eta} \\
= & e^{-k} \sum_{i=0}^{2 \nu} \frac{(2 \nu) !(k+2 \nu)^{2 \nu-1}}{(2 \nu-i) !}
\end{aligned}
$$

The maximum value of above term is $(2 \nu+1) !(k+2 \nu)^{2 \nu}$. Hence from $(2.12)$ we have

$$
E_{R}^{k}(u) \leq C \sqrt{\frac{2}{(2 \nu) !}}\|u(\tau \zeta)\|_{X}\left(\frac{R}{r}\right)^{k}(2 \nu+1)(k+2 \nu)^{2 \nu}
$$

Combining (2.11) with (2.13) we get

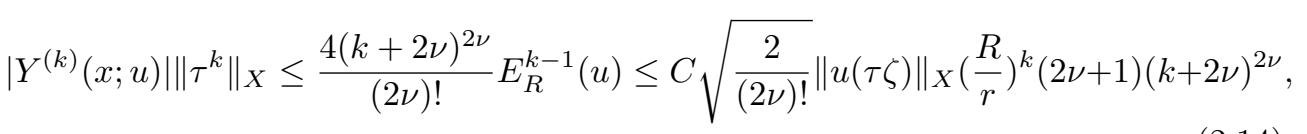

above inequality (2.14) gives the required result.

Lemma 2.3. Let

$$
\alpha_{1}=\liminf _{k \rightarrow \infty}\left(\left\|\tau^{k}\right\|\right)^{\frac{1}{k}} \quad \text { and } \quad \alpha_{2}=\limsup _{k \rightarrow \infty}\left(\left\|\tau^{k}\right\|\right)^{\frac{1}{k}}
$$

Then $\alpha_{1} \geq R$ and $\alpha_{2}<\infty$.

Proof. Suppose $\beta_{k}=\left(\left\|\tau^{k}\right\|\right)^{\frac{1}{k}}$. First we prove that $\alpha_{2}<\infty$. On the contrary we assume that there exists a subsequence $\beta_{k_{m}}$ such that $\lim _{m \rightarrow \infty} \beta_{k_{m}}=\infty$. Consider a function $u_{0}$ such that

$$
u_{0}(\tau x)=\sum_{m=0}^{\infty}\left(\beta_{k_{m}}\right)^{-\frac{k_{m}}{2}} \tau^{k_{m}}
$$

The function $u_{0}(\tau x)$ is entire and therefore belong to $X$. However, in this case using Lemma 2.2 , for any $m \in \mathbb{N}$, we get

$$
\left(\beta_{k_{m}}\right)^{-\frac{k_{m}}{2}}\left\|\tau^{k_{m}}\right\| \leq\left\|u_{0}(\tau x)\right\|<\infty
$$

which is impossible. Hence $\alpha_{2}<\infty$. Now in order to prove $\alpha_{1} \geq R$ we assume that $\alpha_{1}<R$. Set $\delta \in\left(\alpha_{1} ; R\right)$ and consider a function

$$
u_{0}(\tau x)=\sum_{m=0}^{\infty} \delta^{-k_{m}} \tau^{k_{m}}
$$


where $k_{m}$ is a sequence such that $\liminf _{k \rightarrow \infty} \beta_{k}=\lim _{m \rightarrow \infty} \beta_{k_{m}}=\alpha_{1}$. The function $u_{0}(\tau x)$ is harmonic in the ball $B_{\delta}^{n}\left(u_{0}\right), \delta<R$ but not harmonic in ball $B_{R}^{n}$. It is clear that a sequence of partial sums $S_{k, u_{0}}(\tau x)$ of series (2.15) is fundamental in the Banach space $X$ and, therefore, convergence in it to a function $u_{1}(\tau x) \in X$. We now prove that the Fourier-Laplace coefficients of the functions $u_{0}(\tau x)$ and $u_{1}(\tau x)$ are same. For fixed $m \in \mathbb{N} \cup\{0\}, k>m$, we have

$Y^{(k)}\left(x ; u_{1}\right)=Y^{(k)}\left(x ; S_{k, u_{0}}\right)+Y^{(k)}\left(x ; u_{1}-S_{k, u_{0}}\right)=Y^{(k)}\left(x ; u_{0}\right)+Y^{(k)}\left(u_{1}-S_{k, u_{0}}\right)$.

Proceeding to the limit as $k \rightarrow \infty$ and using Lemma 2.2, it gives $Y^{(k)}\left(x ; u_{1}\right)=$ $Y^{(k)}\left(x ; u_{0}\right)$. Hence the function $u_{1}(\tau x) \in X$ but not harmonic in $B_{R}^{n}$, which contradicts the property of the space $X$. Hence $\alpha_{1} \geq R$.

Lemma 2.4. Let $u \in X$ and let $K$ be a compact subset of $\mathbb{R}^{n}, n \geq 3, K \subset B_{R}^{n}$. Then, for $\tau x \in K$,

$$
|u(\tau x)| \leq C\|u(\tau x)\| .
$$

Proof. Let $\gamma=\sup \{|\tau x|: \tau x \in K\}, \gamma<R$. Now write the expansion of $u$ in the Fourier-Laplace series and estimates its modulus by using Lemma 2.2, we get

$$
\begin{gathered}
u(\tau x)=\sum_{k=0}^{\infty} Y^{(k)}(x ; u) \tau^{k} \\
|u(\tau x)| \leq \sum_{k=0}^{\infty}\left|Y^{(k)}(x ; u)\left\|\tau^{k} \mid \leq\right\| u(\tau x)\left\|\sum_{k=0}^{\infty} \frac{\gamma^{k}}{\left\|\tau^{k}\right\|} \leq C\right\| u(\tau x) \|\right.
\end{gathered}
$$

as the series is convergent by Lemma 2.3 .

\section{Main Results}

Theorem 3.1. Let $u \in X$. The condition

$$
\lim _{k \rightarrow \infty}\left(E_{R}^{k}(u)\right)^{\frac{1}{k}}=0
$$

is necessary and sufficient for the function $u$ to be entire.

Proof. Let $u(\tau x)=\sum_{k=0}^{\infty} Y^{(k)}(x ; u) \tau^{k}$ in space $\mathbb{R}^{n}, n \geq 3,0<\tau<R$. In view of Lemma 2.2,

$$
\left|Y^{(k)}(x ; u)\right|\left\|\tau^{k}\right\|_{X} \leq \frac{2 \sqrt{2}(k+2 \nu)}{C \sqrt{(2 \nu) !}(2 \nu+1)(k-1+2 \nu)^{2 \nu}}\left(\frac{r}{R}\right)^{k-1} E_{R}^{k-1}(u)
$$

it gives

$$
\lim _{k \rightarrow \infty}\left|Y^{(k)}(x ; u)\right|^{\frac{1}{k}} \leq \lim _{k \rightarrow \infty}\left(\frac{2 \sqrt{2}(k+2 \nu)}{C \sqrt{(2 \nu) !}(2 \nu+1)(k-1+2 \nu)^{2 \nu}}\left(\frac{r}{R}\right)^{k-1} \frac{E_{R}^{k-1}(u)}{\left\|\tau^{k}\right\|_{X}}\right)^{\frac{1}{k}}=0,
$$


therefore the function $u$ is entire. Now for necessity, from Lemma 2.3, we have

$$
\frac{E_{R}^{k-1}(u) R^{k}}{\left\|\tau^{k}\right\|_{X}} \leq \frac{\sqrt{(2 \nu) !}(2 \nu+1)(k-1+2 \nu)\|u(\tau x)\|_{X} R^{k} r}{2 \sqrt{2}(k+2 \nu)\left\|\tau^{k}\right\|_{X} r^{k}}
$$

Since $u$ is entire and $u \in X$ for any $r>1$, we have

$$
0 \leq \lim _{k \rightarrow \infty}\left(\frac{E_{R}^{k-1}(u) R^{k}}{\left\|\tau^{k}\right\|_{X}}\right)^{\frac{1}{k}} \leq \frac{1}{r} \limsup _{k \rightarrow \infty}\left(\frac{\sqrt{(2 \nu) !}(2 \nu+1)(k-1+2 \nu)\|u(\tau x)\|_{X} R^{k} r}{2 \sqrt{2}(k+2 \nu)\left\|\tau^{k}\right\|_{X}}\right)^{\frac{1}{k}} \leq \frac{1}{r} .
$$

Now by arbitrariness of $r>1$, we obtain

$$
\lim _{k \rightarrow \infty}\left(E_{R}^{k}(u)\right)^{\frac{1}{k}}=0 .
$$

The proof of Theorem 3.1 is completed.

Theorem 3.2. For a function $u \in X$ to be an entire harmonic function in space $\mathbb{R}^{n}, n \geq 3$, of finite order $0<\rho<\infty$, the necessary and sufficient condition is

$$
\limsup _{k \rightarrow \infty} \frac{k \log k}{\log \left(\frac{\left\|\tau^{k}\right\|}{E_{R}^{k}(u)}\right)}=\rho .
$$

Proof. To prove sufficiency part let (3.1) holds therefore the condition of Theorem 3.1 is satisfied and, hence, the function $u$ is entire harmonic in space $\mathbb{R}^{n}, n \geq 3$ and we denote its order by $\rho_{1}$. Thus, on account of Lemma 2.2 we obtain

$$
\rho_{1}=\limsup _{k \rightarrow \infty} \frac{k \log k}{\log \left|Y^{(k)}(x ; u)\right|} \leq \limsup _{k \rightarrow \infty} \frac{k \log k}{\log \left(\frac{\left\|\tau^{k}\right\|}{E_{R}^{k}(u)}\right)}=\rho .
$$

According to the condition of theorem we have to show that $\rho_{1}>0$. On the contrary, we assume that

$$
\limsup _{k \rightarrow \infty} \frac{k \log k}{\log \left|Y^{(k)}(x ; u)\right|}=0
$$

Then for any $\varepsilon, 0<\varepsilon<R$, there exists $K_{\varepsilon}$ such that, for $k>K_{\varepsilon}$

$$
k \log k<-\varepsilon \log \left|Y^{(k)}(x ; u)\right|
$$

or

$$
\left|Y^{(k)}(x ; u)\right|<k^{-\frac{k}{\varepsilon}}
$$

Now for sufficiently large $K_{\varepsilon}$ we have

$$
\left\|\tau^{k}\right\|_{X} \leq\left(\mu_{2}+\varepsilon\right)^{k} \text { and }\left\|\tau^{k}\right\|_{X} \geq(R-\varepsilon)^{k} \text { for } k \geq K_{\varepsilon} .
$$


Then

$$
\begin{aligned}
E_{R}^{k}(u) \leq & \left\|\sum_{j=k+1}^{\infty}\left|Y^{(k)}(x ; u)\right| \tau^{k}\right\|_{X} \leq \sum_{j=k+1}^{\infty} j^{-\frac{j}{\varepsilon}}\left(\mu_{2}+\varepsilon\right)^{j} \\
& \leq \sum_{j=k+1}^{\infty}(k+1)^{-\frac{j}{\varepsilon}}\left(\mu_{2}+\varepsilon\right)^{j}=(k+1)^{-\frac{(k+1)}{\varepsilon}}\left(\mu_{2}+\varepsilon\right)^{k+1}\left(1-\frac{\left(\mu_{2}+\varepsilon\right)}{(k+1)^{\frac{1}{\varepsilon}}}\right)^{-1} .
\end{aligned}
$$

We assume $k+1 \geq\left(\mu_{2}+\varepsilon\right)^{\varepsilon}$ in (3.3), it gives

$$
\frac{\left\|\tau^{k}\right\|_{X}}{E_{R}^{k}(u)} \geq\left(\frac{R-\varepsilon}{\mu_{2}+\varepsilon}\right)^{k+1}(k+1)^{\frac{(k+1)}{\varepsilon}}\left(1-\frac{\mu_{2}+\varepsilon}{(k+1)^{\frac{1}{\varepsilon}}}\right),
$$

or

$$
\log \left(\frac{\left\|\tau^{k}\right\|_{X}}{E_{R}^{k}(u)}\right)^{\frac{1}{k}} \geq\left(\frac{k+1}{k}\right) \log \left(\frac{R-\varepsilon}{\mu_{2}+\varepsilon}\right)+\frac{k+1}{k \varepsilon} \log (k+1)+\frac{1}{k} \log \left(1-\frac{\mu_{2}+\varepsilon}{(k+1)^{\frac{1}{\varepsilon}}}\right)
$$

or

$$
\liminf _{k \rightarrow \infty} \frac{\log \left(\frac{\left\|\tau^{k}\right\|_{X}}{E_{R}^{k}(u)}\right)^{\frac{1}{k}}}{\log k} \geq \frac{1}{\varepsilon},
$$

or

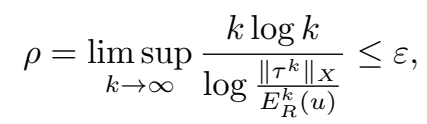

which contradicts our assumption. Now we consider the case for $\varepsilon \in\left(0, \frac{R}{2}\right) \cap\left(0, \rho_{1}\right)$. From the left hand side of (3.2) we conclude that there exists $K_{\varepsilon} \in \mathbb{N}(\varepsilon)$ such that

$$
\left|Y^{(k)}(x ; u)\right|<k^{-\frac{k}{\left(\rho_{1}+\varepsilon\right)}}
$$

for all $k>K_{\varepsilon}$. Let $K_{\varepsilon}$ be sufficiently large such that $\left\|\tau^{k}\right\|_{X} \leq\left(\mu_{2}+\varepsilon\right)$ and $\left\|\tau^{k}\right\| \geq$ $(R-\varepsilon)^{k}$ for $k \geq K_{\varepsilon}$. Then for $k>K_{\varepsilon}$,

$$
\begin{aligned}
E_{R}^{k}(u) \leq \| & \sum_{j=k+1}^{\infty} Y^{(j)}(x ; u) \tau^{j}\left\|\leq \sum_{j=k+1}^{\infty}\left|Y^{(j)}(x ; u)\right|\right\| \tau^{k} \| \\
& \leq \sum_{j=k+1}^{\infty}(j)^{-\frac{j}{\rho_{1}+\varepsilon}}\left\|\tau^{j}\right\| \leq \sum_{j=k+1}^{\infty}(k+1)^{-\frac{j}{\rho_{1}+\varepsilon}}\left(\mu_{2}+\varepsilon\right)^{j} \\
& =\frac{\left(\mu_{2}+\varepsilon\right)^{k+1}}{(k+1)^{\frac{(k+1)}{\left(\rho_{1}+\varepsilon\right)}}}\left(1-\frac{\left(\mu_{2}+\varepsilon\right)}{(k+1)^{\frac{1}{\rho_{1}+\varepsilon}}}\right)^{-1},
\end{aligned}
$$

or

$$
\frac{\left\|\tau^{k}\right\|(k+1)^{\frac{1}{\rho_{1}+\varepsilon}}}{E_{R}^{k}(u)} \geq \frac{\left\|\tau^{k}\right\|}{\left(\mu_{2}+\varepsilon\right)^{k+1}}\left(1-\frac{\left(\mu_{2}+\varepsilon\right)}{(k+1)^{\frac{1}{\rho_{1}+\varepsilon}}}\right)
$$


or

$$
\begin{aligned}
\rho_{1}+\varepsilon \geq & \frac{(k+1) \log (k+1)}{\log \frac{\left\|\tau^{k}\right\|_{X}}{E_{R}^{k}(u)}}\left(1+\frac{\left(\rho_{1}+\varepsilon\right)}{(k+1) \log (k+1)} \log \left(1-\frac{\left(\mu_{2}+\varepsilon\right)}{(k+1)^{\frac{1}{\rho_{1}+\varepsilon}}}\right)+\right. \\
& \left.\frac{\rho_{1}+\varepsilon}{(k+1) \log (k+1)} \log \frac{\left\|\tau^{k}\right\|}{\left(\mu_{2}+\varepsilon\right)^{(k+1)}}\right) .
\end{aligned}
$$

Proceeding to limit as $k \rightarrow \infty$, we get $\rho_{1}+\varepsilon \geq \rho$. Since $\varepsilon$ is arbitrary this implies that $\rho_{1} \geq \rho$. In view of $(3.2)$, we get $\rho_{1}=\rho$, hence the sufficient part is completed. In order to prove the necessary part we assume that $u \in X$ be an entire harmonic function of finite order $\rho$, i.e.,

$$
\limsup _{k \rightarrow \infty} \frac{k \log k}{-\log \left|Y^{(k)}(x ; u)\right|}=\rho .
$$

Set

$$
\rho_{1}=\limsup _{k \rightarrow \infty} \frac{k \log k}{\log \frac{\left\|\tau^{k}\right\|_{X}}{E_{R}^{k}(u)}} .
$$

Here $\rho_{1}$ and $\rho$ are interchanged as compared with the proof of sufficiency part and show that $\rho_{1}=\rho$. By analogy with $(3.2)$, Lemma 2.2 gives $\rho_{1} \geq \rho$. Following the same fact as in the sufficiency part, we can say that, for any $\varepsilon, 0<\varepsilon<R$ there exists $K_{\varepsilon}$ such that

$$
\left|Y^{(k)}(x ; u)\right|<k^{-\frac{k}{(\rho+\varepsilon)}} \text { and }(R-\varepsilon)^{k} \leq\left\|\tau^{k}\right\| \leq\left(\mu_{2}+\varepsilon\right)^{k}
$$

for $k>K_{\varepsilon}$. Following (3.3) and (3.4) (with $\rho_{1}$ and $\rho$ interchanged), we get

$$
\begin{aligned}
\rho+\varepsilon \geq & \frac{(k+1) \log (k+1)}{\log \frac{\left\|\tau^{k}\right\|_{X}}{E_{R}^{k}(u)}}\left(1+\frac{(\rho+\varepsilon)}{(k+1) \log (k+1)} \log \left(1-\frac{\left(\mu_{2}+\varepsilon\right)}{(k+1)^{\frac{1}{\rho+\varepsilon}}}\right)+\right. \\
& \left.\frac{\rho+\varepsilon}{(k+1) \log (k+1)} \log \frac{\left\|\tau^{k}\right\|}{\left(\mu_{2}+\varepsilon\right)^{(k+1)}}\right) .
\end{aligned}
$$

Proceeding to limit as $k \rightarrow \infty$, it gives $\rho \geq \rho_{1}$. Hence the proof is completed.

Theorem 3.3. For a function $u \in X$ to be an entire harmonic function of finite order $\rho \in(0, \infty)$ and normal type $\sigma \in(0, \infty)$, the necessary and sufficient condition is that

$$
\limsup _{k \rightarrow \infty} \frac{k}{e \rho}\left(\frac{E_{R}^{k}(u)}{\left\|\tau^{k}\right\|}\right)^{\frac{\rho}{k}}=\sigma .
$$

Proof. In order to prove sufficiency we assume that $u \in X$ and satisfies the condition of Theorem 3.3 with some positive $\rho$ and $\sigma$. Then (3.1) follows from (3.6), therefore, $u$ is an entire harmonic function of order $\rho$. Assume that the type of $u$ is $T$. We have 
to prove $T=\sigma$. Now using the classical coefficient formula for the type of an entire harmonic function $u \in X$

$$
T=\limsup _{k \rightarrow \infty} \frac{k}{e \rho}\left|Y^{(k)}(x ; u)\right|^{\frac{\rho}{k}}
$$

with Lemma 2.3, we obtain $T \leq \sigma$. To prove the reverse inequality we have from (3.7) that for any $\varepsilon>0$, there exists $K_{\varepsilon} \in \mathbb{N}$ such that, for $k>K_{\varepsilon}$,

$$
\left|Y^{(k)}(x ; u)\right|<\left(\frac{\rho e(T+\varepsilon)}{k}\right)^{\frac{k}{\rho}} .
$$

Following the same techniques as (3.4) and (3.5), we have from (3.8),

$$
E_{R}^{k}(u) \leq \sum_{j=k+1}^{\infty}\left(\frac{\rho e(T+\varepsilon)}{j}\right)^{\frac{j}{\rho}}\left\|\tau^{j}\right\| \leq\left(\frac{\rho e(T+\varepsilon)}{k+1}\right)^{\frac{k+1}{\rho}}(\mu+\varepsilon)^{(k+1)}\left(1-\frac{C^{*}}{(k+1)^{\frac{1}{\rho}}}\right)^{-1}
$$

where $C^{*}=(\mu+\varepsilon)(\rho e(T+\varepsilon))^{\frac{1}{\rho}}$. Now in view of (3.9), we obtain

$$
T+\varepsilon \geq \frac{(k+1)}{e \rho}\left(\frac{E_{R}^{k}(u)}{\left\|\tau^{k}\right\|}\right)^{\frac{\rho}{(k+1)}} \frac{\left\|\tau^{k}\right\|^{\frac{\rho}{(k+1)}}}{(\mu+\varepsilon)^{\rho}}\left(1-\frac{C^{*}}{(k+1)^{\frac{1}{\rho}}}\right)^{\frac{\rho}{(k+1)}} .
$$

Proceeding the limit sup as $k \rightarrow \infty$, we get

$$
T+\varepsilon \geq \sigma\left(\frac{\mu}{\mu+\varepsilon}\right)^{\rho} .
$$

Since $\varepsilon$ is arbitrary and approaches to zero, we get $T \geq \sigma$. Hence the sufficiency part is completed.

Now to prove necessity assume that $u \in X$ is an entire harmonic function of finite order and normal type. We denote its order and type as $\rho$ and $T$ respectively. Further, we have to show that $T=\sigma$. By virtue of (3.7) and Lemma 2.3, we obtain $T \leq \sigma$. Finally, to prove $\sigma \leq T$ repeat the reasoning of sufficiency part. This completes the proof of necessary part. Hence the proof of theorem is completed.

Acknowledgement. The author is extremely thankful to the referees for giving fruitful comments to improve the paper.

\section{References}

[1] H. Beitmen, A. Erdeiy, Vysshye Transtsendentnye Funktsyy, 2nd edition, Nauka, Moscow, 1974, p. 296.

[2] P.L. Duren, B.W. Romberg, A.L. Shields, Linear functionals in $H_{p}$ spaces with $0<p<1$, J. Reine Angew. Math. 238 (1969) 4-60.

[3] A.J. Fryant, Growth of entire harmonic functions in $\mathbb{R}^{3}$, J. Math. Anal. Appl. 66 (1978) 599-605. 
[4] T.B. Fugard, Growth of entire harmonic functions in $\mathbb{R}^{n}, n \geq 2$, J. Math. Anal. Appl. 74 (1) (1980) 289-291.

[5] M.I. Gvaradze, On one class of spaces of analytic functions, Mat. Zametki 21 (2) (1977) 141-150.

[6] G.H. Hardy, J.E. Littlewood, Some properties of fractional integrals, II, Math. Z. 34 (3) (1931) 403-439.

[7] M. Harfaoui, Generalized order and best approximation of entire function in $L_{p^{-}}$ norm, Intern. J. Math.and Math. Sci. 2010 (2010) 1-15.

[8] I.I. Ibragimov, N.I. Shikhaliev, On the best polynomial approximation in one space of analytic functions, Dokl. Akad. Nauk SSSR 227 (2) (1976) 280-283.

[9] I.I. Ibragimov, N.I. Shikhaliev, On the best approximation in the mean for analytic functions in the space $A_{p}(|z|<1)$, Spets. Vopr. Teor. Funkts. 1 (1977) 84-96.

[10] G.P. Kapoor, A. Nautiyal, Approximation of entire harmonic functions in $\mathbb{R}^{3}$, Indian J. Pure and Appl. Math. 13 (9) (1982) 1024-1030.

[11] G.P. Kapoor, A. Nautiyal, On the growth of harmonic functions in $\mathbb{R}^{3}$, Demonstr. Math. 16 (4) (1983) 811-819.

[12] D. Kumar, The growth of harmonic functions in Hyperspheres, Demonstr. Math. 32 (4) (1999) 717-724.

[13] D. Kumar, Growth and approximation of entire harmonic functions in $\mathbb{R}^{n}, n>3$, Georgion Math. J. 15 (1) (2008) 1-12.

[14] D. Kumar, Growth and approximation of solutions to a class of certain linear partial differential equations in $\mathbb{R}^{\mathbb{N}}$, Mathematica Slovaca, 64 (1) (2014) 139154.

[15] D. Kumar, H.S. Kasana, On maximum term, maximum modulus and approximation error of an entire harmonic function in $\mathbb{R}^{3}$, Rev. Mat. Univ. Parma 6 (1) (1998) 215-223.

[16] D. Kumar, H.S. Kasana, Approximation of entire harmonic functions in $\mathbb{R}^{3}$ in $L^{\beta}$-norm, Fasciculi Math. 34 (2004) 55-64.

[17] D. Kumar, G.S. Srivastava, H.S. Kasana, Approximation of entire harmonic functions in $\mathbb{R}^{3}$ having index-pair $(p, q)$, Anal. Numer. Theor. Approx. 20 (1-2) (1991) $47-57$.

[18] A.R. Reddy, A contribution to best approximation in the $L^{2}$-norm, J. Approx. Theory 11 (11) (1974) 110-117.

[19] G.S. Srivastava, Generalized growth of entire harmonic functions, Fasciculi Math. 40 (2008) 79-89. 
[20] G.S. Srivastava, S. Kumar, Uniform approximation of entire functions on compact sets and their generalized growth, New Zealand J. Math. 39 (2009) 33-43.

[21] M. Shaker Abdu-Hussein, G.S. Srivastava, On the generalized type and approximation of entire harmonic functions in $\mathbb{R}^{3}$ having index-pair $(p, q)$, Istanbul Univ. Fem. Fak. Mat. Der. 60 (2001) 1-17.

[22] Y. Stein, H. Veis, , Vvedenye v Harmonycheskyi Analyz na Evklydovykh Prostranstvakh, Moskow, Myr. 1974, p. 336.

[23] A. Tyman, V.N. Trofymov, Vvedenye v Teoryiu Harmony Ches Kykh Funktsii, Nauka, Moscow, 1968, p. 208.

[24] S.B. Vakarchuk, On the best polynomial approximation of analytic functions in the space $B_{p, q, \lambda}$, Dokl. Akad. Nauk Ukr. SSR, Ser. Fiz-Mat. Tekh. Nauk. 8 (1989) 6-9.

[25] S.B. Vakarchuk, S.I. Zhir, , Best polynomial approximations of entire transcendental functions of the generalized order of growth in Banach spaces $\varepsilon_{p}^{\prime}(G)$ and $\varepsilon_{p}(G), p \geq 1$, Ukr. Mat. Vestn. 8 (2) (2011) 255-291.

[26] S.B. Vakarchuk, S.I. Zhir, On the best polynomial approximation of entire transcendental functions of many complex variables in some Banach spaces, Ukr. Math. J. 66 (12) (2015) 1793-1811.

[27] O.V. Veselovskaia, Oroste tselykh harmonycheskykh $v$ funktsyi, Yzv. Vuzov. Matem. 10 (1983) 13-17.

DOI: $10.7862 /$ rf.2021.4

\section{Devendra Kumar}

email: d_kumar001@rediffmail.com

ORCID: 0000-0002-1653-9882

Department of Mathematics, Faculty of Sciences

Al-Baha University

P.O.Box-7738, Alaqiq, Al-Baha-65799

SAUDI ARABIA

Received 11.05.2020

Accepted 30.03.2021 